\title{
AVALIAÇÃO DA DISPERSÃO DE LÍQUIDO EM UM LAVADOR VENTURI UTILIZANDO CFD
}

\author{
A. B. CAMARINHA ${ }^{1}$, R. BÉTTEGA ${ }^{1}$ e V. G. G. BETTEGA ${ }^{1}$ \\ ${ }^{1}$ Universidade Federal de São Carlos, Departamento de Engenharia Química \\ E-mail para contato: vadila@ufscar.br
}

\begin{abstract}
RESUMO - O lavador Venturi é uma alternativa adequada para o crescente interesse em controle de poluição do ar devido a sua eficiência na remoção de partículas numa larga faixa granulométrica. Tal eficiência é influenciada pelas condições operacionais, tais como: a razão líquido/gás, penetração do jato líquido, fração de líquido que se adere às paredes e a distribuição das gotas na garganta. Utilizou-se simulações em CFD por meio do software Fluent a fim de se avaliar o efeito da dimensão e do número de orifícios de injeção de líquido sobre a dispersão de líquido e queda de pressão em um lavador Venturi. Fixando-se a velocidade do gás e a velocidade de líquido injetado em cada orifício, constatouse que maiores orifícios geram jatos de líquido concentrados próximo ao orifício de injeção e influenciam de maneira mais significativa na queda de pressão que o aumento de jatos iguais de líquido, injetados em diferentes pontos de uma mesma seção transversal da garganta.
\end{abstract}

\section{INTRODUÇÃO}

O interesse atual no controle de poluição do ar tem aumentado a importância de avaliarem-se métodos eficientes e econômicos para a remoção de partículas em uma corrente gasosa. Entre os equipamentos utilizados para a limpeza de gases e controle de poluição, o lavador Venturi tem sido uma opção bastante utilizada industrialmente, pois oferece algumas vantagens, nas quais se destaca a sua alta eficiência de remoção de partículas numa larga faixa granulométrica.

O lavador tipo Venturi é basicamente formado por um tubo, de seção transversal circular ou retangular, com uma constrição do tipo Venturi, conforme ilustrado na Figura 1.

Figura 1 - Lavador Venturi típico.

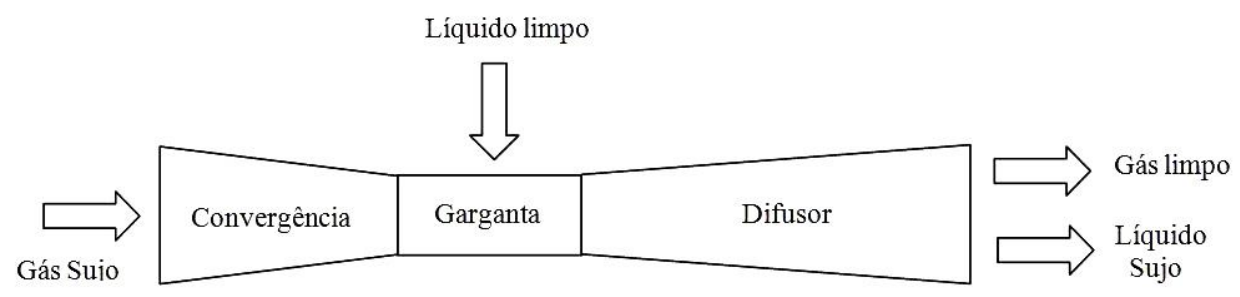

Para a retirada dos contaminantes da corrente gasosa é injetado um líquido de lavagem. Normalmente utiliza-se água, no entanto, pode-se recomendar a utilização de óleos ou 
soluções básicas, dependendo da natureza e afinidade do contaminante. Na saída do lavador Venturi tem-se uma corrente bifásica formada por líquido sujo e gás limpo.

A coleta das partículas em um lavador Venturi é o resultado de mecanismos de coleta que operam simultaneamente: a impactação inercial, a interceptação direta e a difusão. As correlações existentes para predição da eficiência de coleta de partículas em um Venturi envolvem condições operacionais tais como: a razão líquido/gás (L/G), penetração do jato líquido, fração de líquido que se adere às paredes e a distribuição das gotas na garganta (Adelberg, 1967; Azzopardi, 1993; Ananthanarayanan e Viswanathan, 1999; Gonçalves, 2000). Tais condições operacionais influenciam significativamente a eficiência de coleta de partículas de um lavador Venturi. Variações na vazão de líquido de limpeza, vazão do gás e número de orifícios de injeção de líquido afetam a penetração dos jatos no lavador, o processo de atomização, tamanho das gotas, deposição de líquido nas paredes do equipamento e consequentemente a dispersão de gotas em sua garganta (Guerra, 2009). Em 1984 Viswanathan et al. evidenciaram que a distribuição de concentração de gotas foi altamente dependente da razão liquido-gás, mas essencialmente independente da velocidade do ar para um valor fixo de razão líquido-gás.

Em artigo publicado em 2012 Guerra et.al. avaliaram em simulações em CFD utilizando o software Fluent realizadas a partir da implementação de um modelo multifásico VOF o comportamento de múltiplos jatos na injeção de líquido em lavadores Venturi. Os autores evidenciaram que, para mesmo diâmetro de injeção de líquido, o aumento da velocidade do gás (ou sua vazão), com ou sem injeção de líquido, ocasiona o aumento da queda de pressão no lavador. Além disso, confirmaram que o aumento da vazão de líquido, ainda com diâmetro fixo, também causa o aumento da queda de pressão. Já a variação do número de orifícios, mantendo-se fixos o diâmetro de injeção de líquido e a vazão total de líquido, não influenciou de maneira significativa a queda de pressão, porém afeta a distribuição das gotas, o que tem relação direta na eficiência da coleta de partículas.

Assim sendo, o presente estudo teve como objetivo avaliar o efeito da dimensão e do número de orifícios de injeção de líquido sobre a dispersão de líquido e queda de pressão em um lavador Venturi por meio de simulações em CFD utilizando o software Fluent - com abordagem VOF para escoamento multifásico, avaliando diferentes condições operacionais, tais como a velocidade do gás e a vazão de líquido.

\section{METODOLOGIA}

Para realizar as simulações tridimensionais em software Fluent, primeiramente foi necessário construir a malha em software GAMBIT 2.4.6, almejando-se maior refinamento na região da garganta, principalmente no local da injeção de líquido pelos orifícios, pois representa a região de maior turbulência e interação entre as fases líquida e gasosa. A Figura 2 mostra um esquema representativo do lavador Venturi construído e a Tabela 1 apresenta suas principais dimensões. Os orifícios de injeção do líquido, quatro no total, encontram-se distribuídos um em cada parede da garganta do lavador. Para a avaliação da influência do diâmetro do orifício de injeção de líquido, foram adotados valores de diâmetros de injeção iguais a 0,$7 ; 1$ e 1,3 mm. Os casos simulados estão apresentados na Tabela 2. 
Após a construção da malha, as simulações numéricas tridimensionais em software Fluent 14.5 foram feitas utilizando-se abordagem Euleriana em um modelo multifásico (VOF), o qual é baseado no fato de que as fases não são interpenetráveis. A corrente foi considerada incompressível e isotérmica com duas fases, gasosa (ar) e líquida (água). Para a solução numérica, foi utilizada a configuração: escoamento turbulento com modelo de resolução numérico $\mathrm{k}-\varepsilon \mathrm{RNG}, 1^{\mathrm{a}}$ ordem para a quantidade de movimento e QUICK para a fração volumétrica.

Figura 2 - Esquema do lavador Venturi construído em (a) vista de cima e (b) vista lateral.

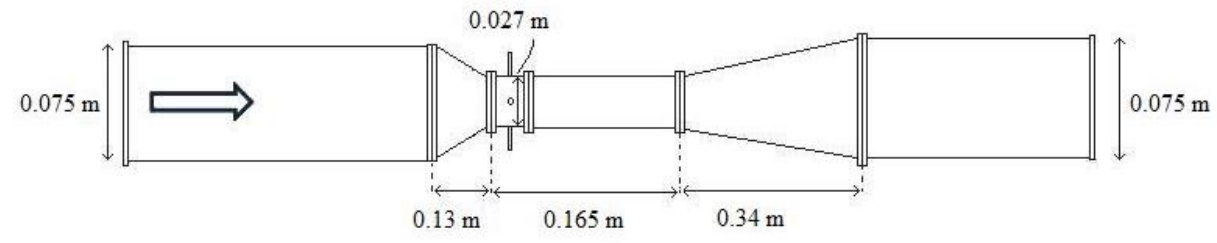

(a)

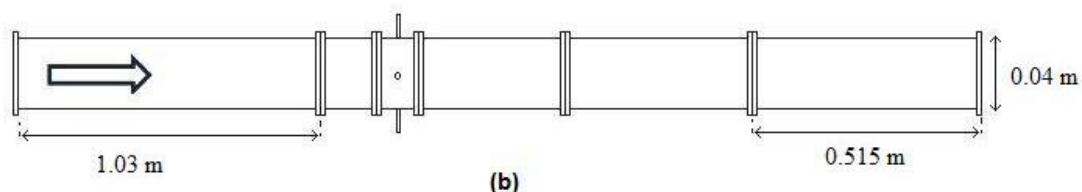

(b)

Tabela 1 - Características da malha utilizada nas simulações em CFD.

\begin{tabular}{cc} 
Grandeza & Valor $(\mathbf{m})$ \\
\hline Altura & 0.040 \\
Largura na entrada e saída & 0.075 \\
Largura da garganta & 0.027 \\
Comprimento da seção convergente & 0.130 \\
Comprimentos de garganta & 0.165 \\
Comprimento da seção divergente & 0.340 \\
Meio ângulo convergente & $10,5^{0}$ \\
Meio ângulo divergente & $4,0^{0}$ \\
\hline
\end{tabular}

Tabela 2 - Identificação dos diferentes casos simulados.

\begin{tabular}{cc|cc|cc}
\multicolumn{7}{c}{ Diâmetro $(\mathbf{m m})$} \\
\hline \multicolumn{2}{c|}{0,7} & \multicolumn{2}{c}{1,0} & \multicolumn{2}{c}{1,3} \\
\hline Caso & Orifícios & Caso & Orifícios & Caso & Orifícios \\
\hline $1-1$ & 1 & $2-1$ & 1 & $3-1$ & 1 \\
$1-2$ & 2 & $2-2$ & 2 & $3-2$ & 2 \\
$1-3$ & 3 & $2-3$ & 3 & $3-3$ & 3 \\
$1-4$ & 4 & $2-4$ & 4 & $3-4$ & 4 \\
\hline
\end{tabular}




\section{RESULTADOS E DISCUSSÃO}

A malha resultante possui 336.879 células e foi construída em blocos de volumes para a entrada, as regiões de convergência e divergência, a garganta e a saída. Em sua grande parte, é constituída de células hexaédricas, exceto na região da garganta, principalmente no local dos bicos de injeção de líquido, onde se apresenta como uma região não-estruturada. Foi dado maior atenção aos bicos de injeção, por possuírem formato circular. Neles, foram impostos formatos de células que acompanhassem os anéis circulares, garantido, dessa forma, maior número de células nessa região. Dessa forma, a garganta do lavador, região de maior interesse, apresentou maior número de células e maior refino de malha. Nas Figuras 3 e 4, é ilustrada a malha construída e uma aproximação demonstrando a montagem dos orifícios, possibilitando a variação do diâmetro na posterior simulação.

\section{Figura 3 - Malha construída em software GAMBIT 2.4.6.}

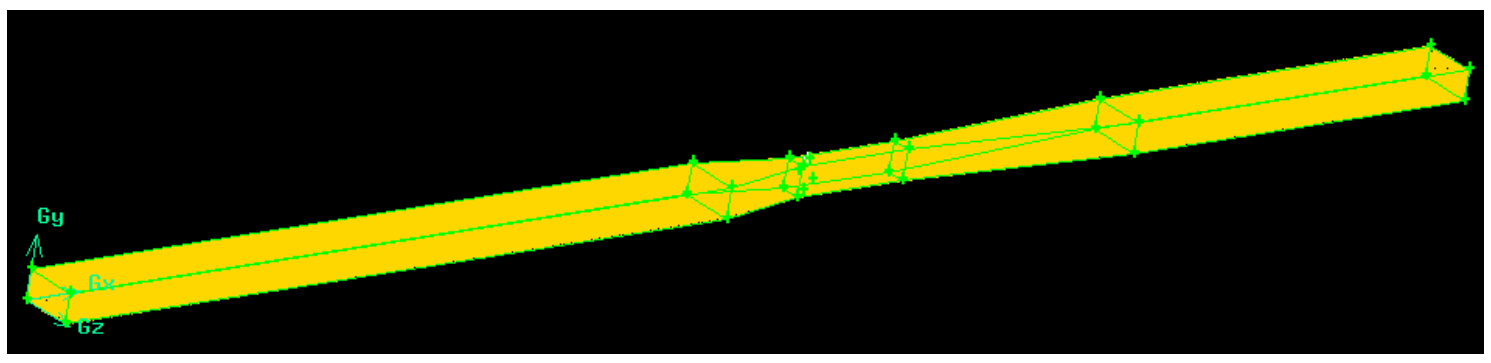

Figura 4 - Aproximação de imagem na seção inicial da garganta.

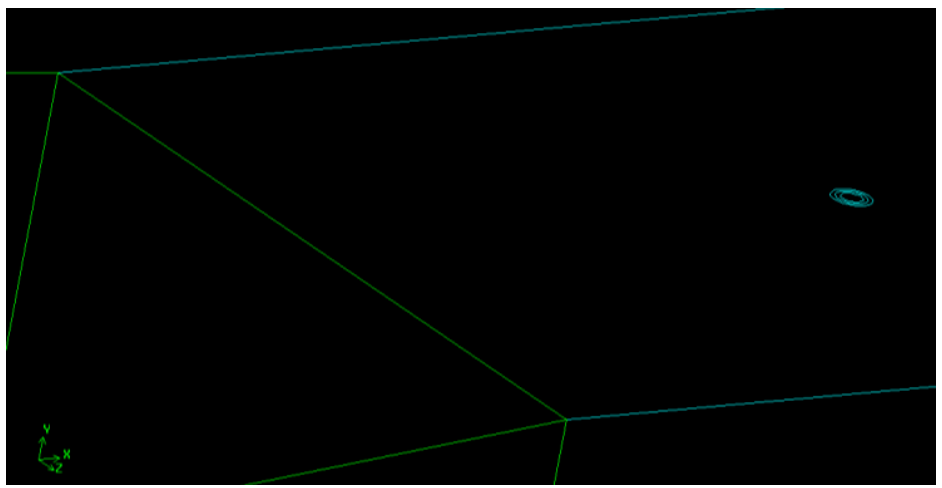

Para avaliar a influência da dimensão do diâmetro na queda de pressão $(\Delta \mathrm{P})$ e na distribuição das gotas, foi necessário fixar alguns parâmetros. Em um primeiro caso, fixou-se

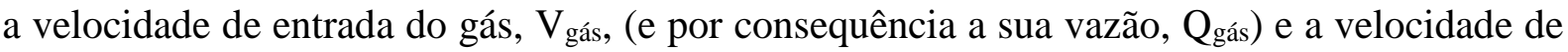
injeção de líquido, $V_{\text {líq }}$ em cada orifício em 23 e 9,7 m/s, respectivamente. Dessa forma, a vazão de líquido, Qlíq, aumenta com o aumento do número e do diâmetro dos orifícios. Simulada a malha de acordo com as configurações e condições operacionais supracitadas, foram obtidos os resultados ilustrados na Figura 5 e na Tabela 3.

A Figura 5 mostra a penetração do jato na garganta do lavador Venturi para diferentes números e diâmetros de orifícios de injeção de líquido. Como pode ser observado ao entrar na garganta do equipamento o jato é arrastado pela corrente de ar a alta velocidade e sofre uma curvatura. Como pode ser visto na Figura 5 a vazão de líquido, número e diâmetro dos 
orifícios de injeção influenciam significativamente na distribuição do líquido no interior da garganta do lavador. A uniforme distribuição e líquido favorece a eficiência de coleta de partícula do equipamento.

Figura 5 - Contornos de fração de fase para todos os casos avaliados.
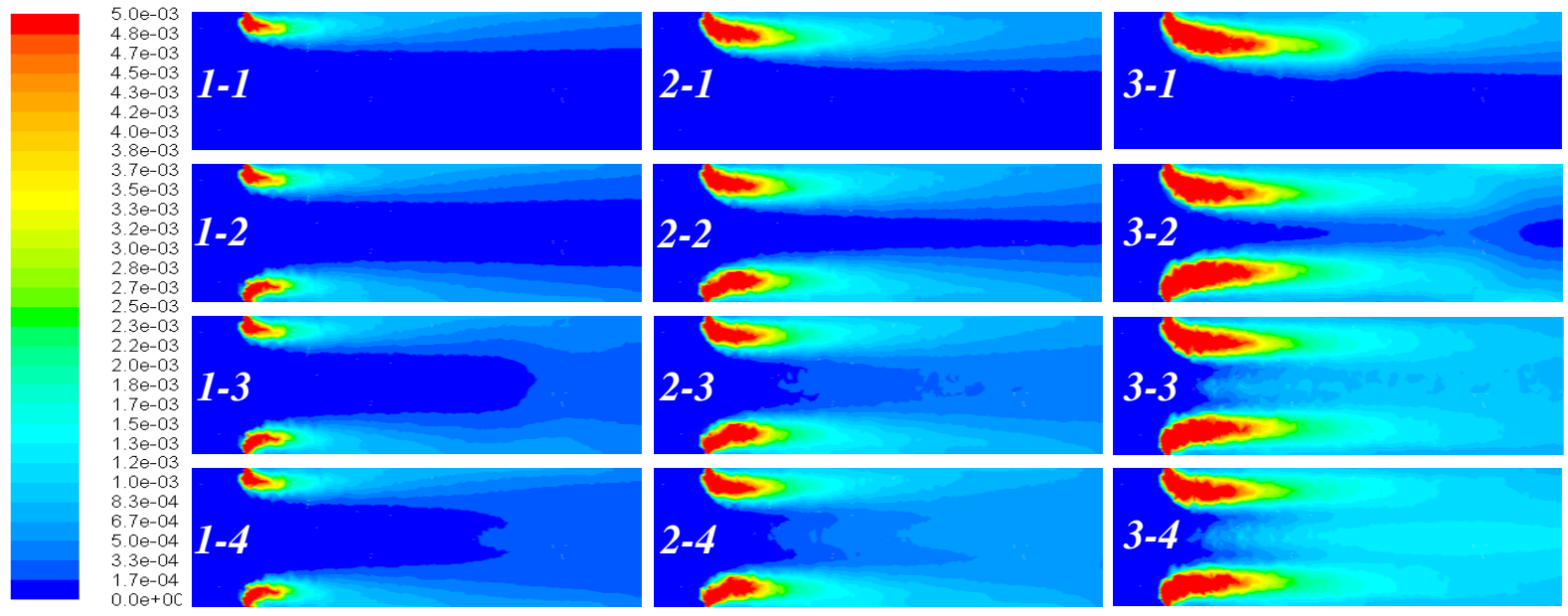

Tabela 3 - Valores obtidos de queda de pressão, $\Delta \mathrm{P}$, e vazão de líquido, Qlíq, para diferentes diâmetros, fixando-se a velocidade do gás e a velocidade de líquido em cada orifício.

\begin{tabular}{|c|c|c|c|c|c|c|}
\hline \multirow[b]{3}{*}{$\begin{array}{c}\mathbf{N}^{\circ} \\
\text { Orifícios }\end{array}$} & \multicolumn{6}{|c|}{ Diâmetros de injeção de líquido (mm) } \\
\hline & \multicolumn{2}{|r|}{0,7} & \multicolumn{2}{|r|}{1,0} & \multicolumn{2}{|r|}{1,3} \\
\hline & $\begin{array}{c}\Delta \mathbf{P} \\
(\mathbf{P a})\end{array}$ & Qlíq (mL/min) & $\begin{array}{c}\Delta \mathbf{P} \\
(\mathbf{P a})\end{array}$ & Qlíq (mL/min) & $\begin{array}{c}\Delta \mathbf{P} \\
(\mathbf{P a})\end{array}$ & Qlíq (mL/min) \\
\hline 1 & 864,5 & 224,0 & 1004,4 & 457,1 & 1306,4 & 772,5 \\
\hline 2 & 985,6 & 448,0 & 1260,9 & 914,2 & 1897,1 & 1545,0 \\
\hline 3 & 1035,8 & 671,9 & 1545,4 & 1371,3 & 2199,6 & 2317,5 \\
\hline 4 & 1245,8 & 895,9 & 2082,8 & 1828,4 & 2607,8 & 3090,0 \\
\hline
\end{tabular}

Como esperado, observa-se pela Tabela 2 que a queda de pressão se eleva com o aumento do número de orifícios para um mesmo diâmetro e com o aumento do diâmetro para um mesmo número de orifícios. Isso ocorre devido ao fato de se injetar maior volume de líquido em igual vazão de gás, o que demanda uma maior quantidade de energia para o arraste do líquido.

Ainda pela Tabela 3, nota-se que o aumento do número de orifícios ocasiona uma menor elevação da queda de pressão em comparação ao aumento do diâmetro, sendo observado um acompanhamento em valores da vazão de líquido. Dessa forma, a explicação citada anteriormente também se aplica a estes casos.

No entanto, em uma comparação mais extrema, nota-se que, partindo-se de 1 orifício e diâmetro $0,7 \mathrm{~mm}$, seria mais vantajoso em relação a queda de pressão quadriplicar o número de orifícios, e por consequência a vazão de líquido (Q líq $_{1}=895,9 \mathrm{ml} / \mathrm{min}$.), que aumentar o diâmetro para 1,3 (Q líq $_{1} 772,5 \mathrm{ml} / \mathrm{min}$.). Neste caso, aquele que apresentou maior vazão de 
líquido obteve uma menor queda de pressão. Isso aponta a possibilidade de que maiores corpos de líquido (jatos), concentrados próximo ao orifício de injeção, influenciam de maneira mais significativa na queda de pressão que o aumento do número de jatos líquido injetados em diferentes pontos de uma mesma seção transversal da garganta.

Além de apresentar menor queda de pressão, esse aumento do número de orifícios se mostra interessante em termos de distribuição de líquido na garganta do equipamento e consequentemente possibilidade de melhora na coleta de partículas, devido ao fato de se operar com mais pontos de injeção de líquido ao redor da garganta, com a mesma capacidade de penetração do jato, pois todos os orifícios possuem a mesma velocidade de injeção de líquido.

\section{CONCLUSÕES}

A simulação de um lavador Venturi fixando-se a velocidade de gás na entrada e a velocidade de líquido de injeção em cada orifício permitiu a detecção de uma vantagem em termos de queda de pressão ao aumentar-se o número de orifícios em comparação ao aumento da dimensão do diâmetro de injeção de líquido, levantando-se a possibilidade de que maiores corpos de líquido concentrados próximo ao orifício influenciam de maneira mais significativa na queda de pressão que o aumento de corpos iguais de líquido injetados em diferentes pontos de uma mesma seção transversal da garganta.

\section{REFERÊNCIAS BIBLIOGRÁFICAS}

ADELBERG, M. Break-up rate and penetration of a liquid jet in a gas stream. AIAA Journal,v.5, p.1408-1415, 1967.

ANANTHANARAYANAN, N. V.; VISWANATHAN, S. Predicting the liquid flux distribution and collection efficiency in cylindrical Venturi scrubber. Ind. Eng. Chem. Res., v. 38, p. 223-232, 1999.

AZZOPARDI, B.J. Liquid distribution in Venturi scrubbers: the importance of liquid films on the channel walls. Chem. Eng. Sci., v.48, n.15, p.2807-2813, 1993.

GONÇALVES, J.A.S. Aspectos da modelagem matemática de lavadores Venturi. Tese de doutorado. Universidade Federal de São Carlos, São Carlos - Brasil, 2000.

GUERRA, V.G. Investigação do efeito de injeção de líquido por orifícios múltiplos na formação de gotas em um lavador Venturi. Universidade Federal de São Carlos, São Carlos - Brasil, 2009.

GUERRA, V.G.; BETTEGA, R.; GONÇALVES, J.A.S.; COURY, J.R. Pressure Drop and Liquid Distribution in a Venturi Scrubber: Experimental Data and CFD Simulation, Ind. Eng. Chem. Res.,v.51, p 8049-8060, 2012.

VISWANATHAN, S.; GNYP, A.W.; ST. PIERRE, C.C. Examination of gas flow in a Venturi scrubber. Ind. Eng. Chem. Fund., v.23, p.303-308, 1984. 